\title{
Effect of Automated Immunization Registry-Based Telephonic Interventions on Adult Vaccination Rates in Community Pharmacies: A Randomized Controlled Trial
}

\author{
Samuel Stolpe, PharmD, MPH, and Niteesh K. Choudhry, MD, PhD
}

\begin{abstract}
BACKGROUND: Pharmacies have a unique opportunity to address suboptimal adult vaccination rates, but few solutions have proven effective. Such strategies are challenged by the lack of access that many pharmacies have to a patient's complete immunization history; consequently, they are unable to identify which of their patients actually require vaccination. A pharmacybased strategy that leverages such information could enhance efforts to increase rates of guideline-based vaccination.
\end{abstract}

OBJECTIVE: To determine the effect on vaccination rates of an automated telephonic intervention for adults in need of either pneumococcal vaccination or herpes zoster vaccination, or both.

METHODS: Over a 1-year period, patients with identified vaccine gaps at 246 pharmacies of 3 pharmacy chains were randomly assigned to receive either usual care or an automated telephonic prompt for pneumococcal and/or herpes zoster vaccines based on patient records contained in state immunization registries and pharmacy data. The primary outcome was the proportion with administration of at least one of the vaccines offered between March 2016 and January 2017 based on intention-to-treat principles. Subgroup analyses included vaccination rates by age and sex. An as-treated analysis was also performed.

RESULTS: 21,971 patients were included in the study, $57 \%$ of whom were female, with a mean age of 63 years. Vaccine administration proportions were $0.0214(236 / 11,009)$ in the intervention group, and 0.0205 $(225 / 10,962)$ in the control group $(\mathrm{OR}=1.05,95 \% \mathrm{Cl}=0.87-1.26)$. Results did not differ in subgroup analyses based on patient age, sex, or individual pharmacy chain. Among intervention patients, 3,666 (0.333) completed the call by listening to the entire prompt. In an as-treated analysis comparing individuals who completed calls versus control, the intervention increased the odds of vaccination by $26 \%(0 \mathrm{R}=1.26,95 \% \mathrm{Cl}=1.00-1.61)$.

CONCLUSIONS: The automated prompt did not significantly increase vaccination rates. Potential barriers included intervention technical flaws, low rates of connecting with patients, insufficient follow-up by the pharmacy, and patients placing a relatively low priority on being vaccinated.

J Manag Care Spec Pharm. 2019;25(9):989-94

Copyright $\odot 2019$, Academy of Managed Care Pharmacy. All rights reserved.

\section{What is already known about this subject}

Automated direct telephonic outreach from pharmacies has been shown to modestly improve vaccination rates.

Query of immunization information systems (IIS) to obtain immunization histories and forecast needed vaccines is a recognized best practice for identification and closure of immunization gaps.

While use of IIS within community pharmacy practice is still in a nascent stage, early research has shown improvements in vaccination rates for the adult population when pharmacists have access to IIS histories at the point of care.

\section{What this study adds}

This study is the first randomized controlled trial that explored using an automated telephonic prompt based on vaccination gaps identified in the IIS to persuade patients to receive a vaccine as a message added at the end of a regularly occurring call.

This study suggests that vaccine-specific messaging is not likely to be acted on by patients when appended to another telephonic message

The study also suggests the importance of modality selection in prompting patients for pharmacy immunization services.

T he development of safer and more effective vaccines over the past century is widely considered one of the most important advances of modern medicine, particularly because of its positive effects on public health. ${ }^{1-3}$ Despite this, among older adults only $66 \%$ get the annual flu vaccine; approximately $60 \%$ are immunized against pneumococcal disease; and $20 \%$ are immunized against herpes zoster-well below public health goals of $90 \%, 90 \%$, and $30 \%$, respectively. ${ }^{4,5}$ As a result, vaccine-preventable illness and hospitalizations continue to occur at rates much higher than necessary. For example, the vaccination of $42 \%$ of Americans during 2017-2018 resulted in an estimated prevention of 7.1 million illnesses, 3.7 million medical visits, 109,000 hospitalizations, and 8,000 deaths associated with flu. ${ }^{6}$ In the U.S. adult population, vaccine-preventable illness was estimated in 2016 to occur at over 18,500,000 cases per year. The associated net economic burden is nearly $\$ 9$ billion, over $90 \%$ of which was attributed to poor vaccination rates. ${ }^{7}$ 
There are many reasons for under-vaccination among adult patients. The most common reasons cited by patients and providers include lack of vaccine recommendations to patients from health care providers, inadequate reminder systems, and poor understanding and faulty assumptions on the part of patients. ${ }^{8}$ Many patients indicate a willingness to receive recommended vaccines but report low rates of health care providers putting those recommendations forward. ${ }^{9}$

Pharmacist authorization to administer vaccines has helped address some of the reasons for under-vaccination among adults. Accompanying the expansion of pharmacist practice authority to include vaccinations has been a significant increase in the number of patients who receive their vaccines in community pharmacies. According to Centers for Disease Control and Prevention (CDC) survey data, pharmacists administered $6 \%$ of total influenza vaccinations in the 2005-2006 flu season but 25\% in the 2015-2016 season. ${ }^{10,11}$ Despite this growth, community pharmacies do not have a comprehensive view of patients' immunization history and so are challenged to efficiently identify additional vaccination needs beyond influenza.

Access to state immunization registries (also called "immunization information systems" or "IIS") in everyday workflows could improve identification and closure of vaccination gaps, but as of yet, IIS-based automated outreach to patients offering needed vaccines is not part of commonplace community pharmacy practice and has never been researched. Therefore, we tested a novel automated telephonic vaccination prompt based on IIS histories to invite patients to receive missing vaccinations.

\section{Methods}

\section{Study Design}

The Immunization Services Model for Adult Rate Improvement (ImmuSMART) study was a randomized controlled experiment that examined the effect on adult vaccination rates of a prompt to receive a pneumococcal and/or herpes zoster vaccination for patients that fit vaccine recommendation criteria for age or high-risk status according to the CDC's Advisory Council on Immunization Practices (ACIP) guidelines. ${ }^{12}$ ImmuSMART was conducted as a collaboration between the Pharmacy Quality Alliance, Scientific Technologies Corporation, VoicePort, and 246 stores of 3 community pharmacy chains in New York, Pennsylvania, and Vermont.

The study was approved by the Chesapeake Institutional Review Board and was granted exemption from informed patient consent. ImmuSMART was listed on the ClinicalTrials. gov trial registry (Identifier: NCT02609035).

\section{Patient Eligibility and Enrollment}

All adult patients aged 19 years and older who were scheduled to receive an automated telephone call from their community pharmacies were potentially eligible for inclusion. The nature of these routinely scheduled calls varied by pharmacy chain.
Potentially eligible patients were those receiving a medication synchronization pre-appointment call at 1 chain (100 stores), a refill reminder call at the second chain (88 stores), and a refill ready call at the third chain (58 stores).

Subjects were included if on the day before the scheduled call, they met 1 or both study eligibility criteria: (a) individuals who were missing a pneumococcal vaccination and were aged either at least 65 years or between 19 and 64 years with potentially high-risk conditions and (b) individuals aged at least 60 years who were missing a herpes zoster vaccination. There were no study exclusion criteria.

Data from IIS and pharmacy-dispensing histories were used to identify missing study vaccinations. Dispensing data were also used to identify potentially high-risk patients by National Drug Code identifiers associated with medications used to treat diabetes, heart failure, asthma, chronic obstructive pulmonary disease, immunosuppression, smoking, or alcoholism. These conditions are considered high-risk indicators for pneumococcal vaccination by current ACIP guidelines. ${ }^{12}$ According to annual reports produced by the $\mathrm{CDC}$, the records contained in the IIS for adults varies by state, with national participation at $44 \%$. During this study of pharmacies in New York, Pennsylvania, and Vermont, adult participation in their state IIS was 19.3\%, $48.7 \%$, and $73.7 \%$, respectively. ${ }^{13}$ Participation indicated only that the patient had at least 1 vaccine in the IIS and was not correlated with record completeness. Missing a study vaccine in a patient's record within either the IIS or the dispensing data was considered an eligibility criterion for the study.

Enrollment was scheduled to begin in August 2014, but because of delays in development of the technology deployed in the study, enrollment began on March 31, 2015, and continued through January 5, 2016. An influenza vaccine intervention initially included in the study was removed as a consequence of missing recruitment during the 2014-2015 influenza season.

\section{Randomization and Intervention}

Once a vaccination gap was identified for the patient in the IIS and pharmacy-dispensing data, patients were randomly assigned in a 1:1 ratio to intervention or control. Randomization was stratified by pharmacy chain. The randomization code was prepared using computer-generated random numbers, with patients having equal probability of being assigned to either group.

For intervention patients, a set of automated scripts offering the vaccines under study were developed by VoicePort and approved by the study principal investigator (Appendix A, available in online article). The vaccination prompt was appended to the outbound communication that patients were scheduled to receive and offered either pneumococcal vaccine, herpes zoster vaccine, or both. Two additional attempts were made if the patient did not answer the phone, if the call went to an answering machine, or if the patient ended the call 


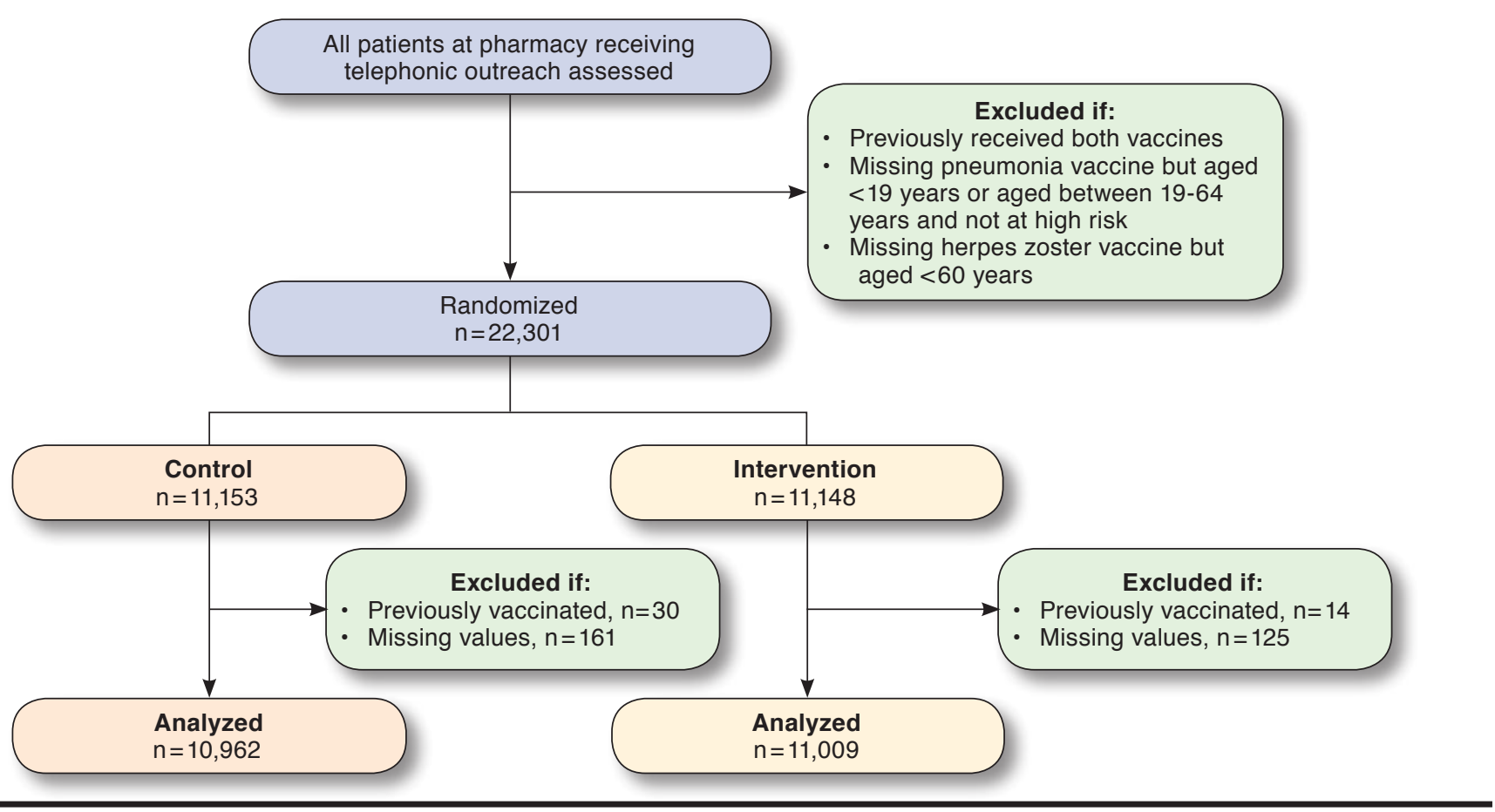

before receiving the vaccination prompt. Patients who listened to the entire vaccination prompt were asked to give a vocal response indicating their intent to receive the vaccine during their next visit to the pharmacy. If the patient indicated acceptance, a notification appeared within the pharmacy's clinical platform with an alert generated for the pharmacist indicating the patient's response. No further outreach to the patient was defined in the study protocol.

Patients in the control group received their scheduled outbound communication but without the added vaccination prompt.

\section{Study Outcomes}

The primary outcome of this study was the proportion with administration of at least 1 of the vaccines offered via the automated telephonic prompt between March 2015 and January 2016. Patients who were offered 2 vaccines met the outcome definition if only 1 of the 2 was administered. The rate was determined by pharmacy-dispensing data alone, not in combination with IIS data.

Secondary outcomes for this study assessed patient receptiveness to vaccination prompts. To this end, call data were used to analyze the rate at which calls were completed, with a completed call defined as a patient listening to the entire vaccination prompt. The rate that patients responded to the prompt was also analyzed.

\section{Statistical Analysis}

Because of a lack of access to baseline data, we conservatively assumed a vaccination rate of 0.50 in the control group. A desired absolute risk reduction of 0.025 was chosen, assuming that a smaller reduction in risk would not offer sufficient incentive for pharmacies to adopt the technology. At a 2-sided alpha-level of 0.05 and a power of 0.90 , a total sample size of 16,798 patients was required to adequately power the study.

Descriptive statistics were used to summarize the baseline characteristics of the study population and the proportion of intervention subjects that listened to the vaccination prompt. Intervention and control subjects were compared using the independent t-test for continuous variables and the chi-square test for categorical variables.

The primary analyses were performed using intention-totreat principles and were conducted using unadjusted logistic regression. The models were re-run, adjusting for known influencers of vaccination rates, including age and sex, as well as ZIP code-level median income, race (percentage African American), and educational level (percentage undergraduate or higher complete). All covariates in the modeling were continuous variables with the exception of sex as a categorical variable. Subgroup analyses were performed for age and sex, with age split into 2 subgroups of $<60$ and $\geq 60$. An as-treated analysis was also performed comparing controls with those intervention subjects who completed a call. 


\begin{tabular}{|c|c|c|}
\hline \multirow[b]{2}{*}{ Characteristics } & \multicolumn{2}{|c|}{ Randomization Assignment } \\
\hline & Control & Intervention \\
\hline Number & 10,962 & 11,009 \\
\hline Age, years, mean (SD) & $63.3 \quad(14.1)$ & $63.2 \quad(14.0)$ \\
\hline Female, \% & 57.7 & 56.9 \\
\hline $\begin{array}{l}\text { Median income in ZIP code, } \\
\text { mean (SD), \$ }\end{array}$ & $67,079(17,034)$ & $67,019(17,135)$ \\
\hline Black race in ZIP code, mean (SD), \% & $(0.1)$ & $(0.1)$ \\
\hline $\begin{array}{l}\text { Education in ZIP code, } \\
\% \text { undergrad or higher (SD) } \\
\end{array}$ & $26.8 \quad(12.5)$ & $26.6 \quad(12.3)$ \\
\hline
\end{tabular}

\section{Results}

Between March 2015 and January 2016, a total of 22,301 patients were randomized to intervention (Figure 1). A total of $44(0.2 \%)$ subjects were removed from the analysis because they had received a vaccine before randomization. In addition, $268(1.3 \%)$ subjects were missing sociodemographic data and were also excluded.

The baseline characteristics were well balanced between the control and intervention groups (Table 1). The average age was 63 years, and approximately 57\% of the patients were women. ZIP code-level mean income, educational attainment, and race distribution were similar in the 2 study groups.

Analysis of call completion data among intervention subjects showed that $28.2 \%$ of first call attempts were completed. Second and third attempts had completeness rates of $6.2 \%$ and $1.0 \%$, respectively, resulting in a study call completeness rate of $33.3 \%$. Patients who completed the calls were older, more likely to be female, and more likely to live in areas with lower incomes, lower education, and less racial diversity than intervention subjects (Appendix B, available in online article).

During follow-up, 236 (2.14\%) intervention patients and $225(2.05 \%)$ control patients were vaccinated (odds ratio $[\mathrm{OR}]=1.05$; 95\% confidence interval $[\mathrm{CI}]=0.87-1.26$; Table 2). Results were virtually identical in the crude and adjusted models and did not differ within age or sex subgroups. Vaccination rates by the specific vaccine offered are shown in Figure 2.

The results of the as-treated analysis are presented in Table 3. Intervention subjects who completed the call were more likely to get vaccinated than controls (unadjusted $\mathrm{OR}=1.36 ; 95 \%$ $\mathrm{CI}=1.08-1.73$ ). Models controlling for age, sex, income, education attainment, and race produced similar results $(\mathrm{OR}=1.30$; $95 \% \mathrm{CI}=1.00-1.61)$.

\section{Discussion}

In this randomized controlled trial that analyzed 21,971 patients who received a regular automated telephone outreach from their pharmacy, appending an additional message based on apparent gaps in their immunization history as a prompt to receive the

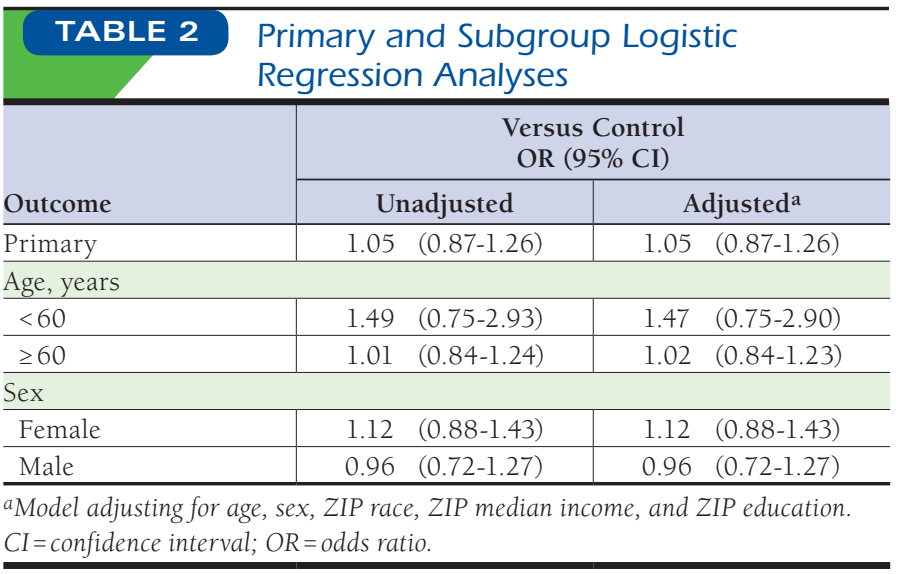

missing vaccines did not significantly increase adult vaccination rates. The as-treated analysis suggests that patients who listened to the calls completely were more likely to be vaccinated, with the limitation that health behaviors of such patients may differ from those of the general population. This offers some evidence that the intervention may be effective were it more efficient in connecting with patients, either as a stand-alone call or through a different modality such as text messaging.

Previous studies conducted in community pharmacies have tested a variety of methods to improve vaccination rates among adults. ${ }^{14}$ These studies have found that passive methods to promote vaccines in the pharmacy, such as placement of signage and educational materials, are less effective than direct, personal communication vehicles, such as educational interventions, direct conversations with patients, and personalized letters. ${ }^{15-18}$

Automated telephonic outreach has also been tested, with modest but significant effects in herpes zoster vaccine uptake seen from a stand-alone 30 -second outbound message from the pharmacy. ${ }^{19}$ Our concern about such an outreach method was that patients might view it as an unsolicited marketing effort on the part of the pharmacy rather than as a medical best practice performed in the interests of the patient. We theorized that appending the vaccination prompt to a message that the patient had given consent to receive would offset some of the potential for this perception.

\section{Limitations}

Our study was limited by the efficiency of telephone calls for outreach. The relatively low call completion rate of 33\% is indicative of inefficiencies in the modality overall. Many calls simply were not picked up by the intended recipient or went to voicemail. Moreover, for the calls that were picked up, most were not listened to in their entirety. Placing the vaccination prompt at the end of the message made it more likely that the patient would not receive it. Hence, most intervention 


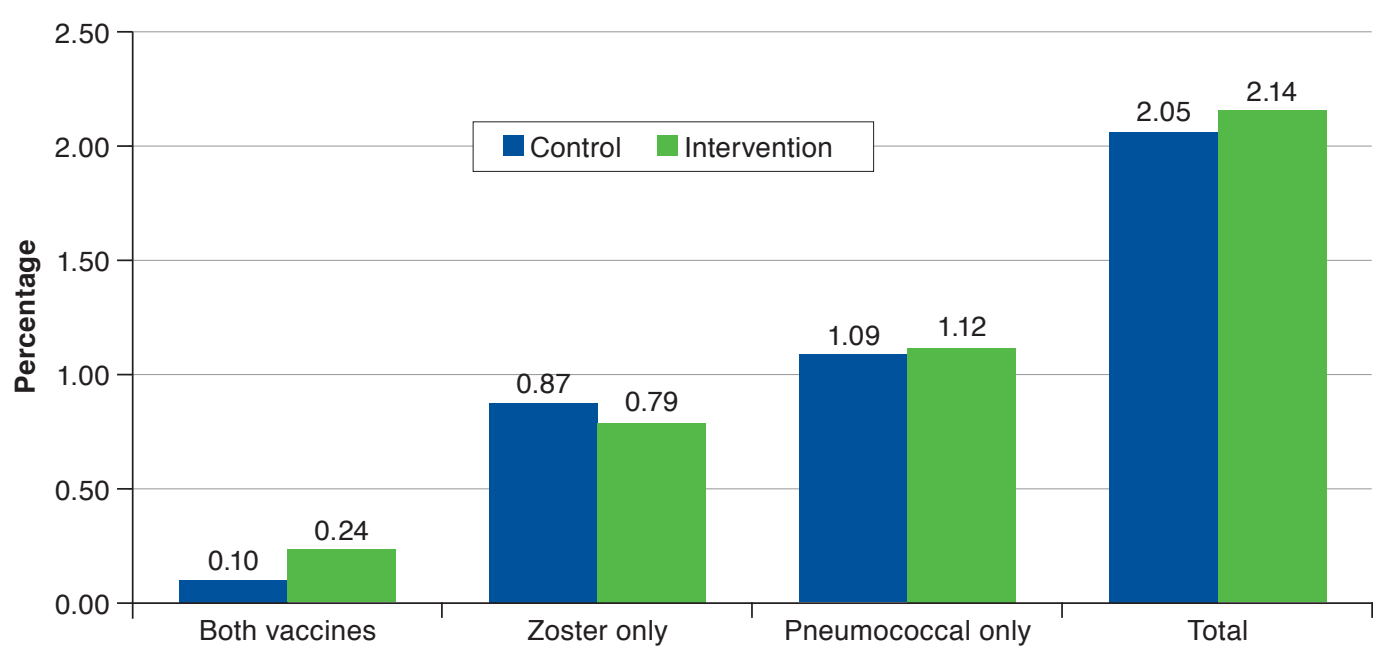

patients often did not receive the vaccine prompt at all. The low engagement rate for our study was counterbalanced by the large sample size of patients. Nonetheless, text and mobile calls would likely be more efficient outreach vehicles. This represents another area for potential research.

Several other limitations of our study and the intervention should be acknowledged. Site differences and site variability in the types of the outbound messages that served as enrollment and randomization triggers were potential limitations. These were counterbalanced by the general uniformity of pharmacy practice across community practice settings.

Because study sites were located in states with variable and generally incomplete adult participation in the immunization registries, this may have resulted in a bias toward the null hypothesis. Falsely identified missing vaccines resulting from incomplete registry data would result in offering vaccines to patients who have already received them, and such patients would likely not be vaccinated again.

In addition, our assessment of the outcome may have been affected by missing data. Because we only assessed the outcome with pharmacy data, we did not know if patients received intervention vaccines at a health care setting other than their community pharmacy. This would also bias the result toward the null.

\section{Conclusions}

The use of automated immunization registry-based telephonic prompts to patients as an appended message to regularly occurring outbound calls did not improve adult rates of pneumococcal and herpes zoster vaccination. This is likely because of limitations stemming from intervention flaws in the modality itself and in technological design and implementation. Low

\section{TABLE 3 Logistic Regression As-Treated Analysis}

\begin{tabular}{l|c|c}
\multirow{2}{*}{ As-Treated Outcome } & \multicolumn{2}{|c}{$\begin{array}{c}\text { Versus Control } \\
\text { OR (95\% CI) }\end{array}$} \\
\cline { 2 - 3 } Primary & Unadjusted & Adjusted $^{\mathrm{a}}$ \\
\hline
\end{tabular}

${ }^{a}$ Model adjusting for age, sex, ZIP race, ZIP median income, and ZIP education. $C I=$ confidence interval; $O R=$ odds ratio.

rates of connecting with patients, poor adult data in the state IIS, and insufficient pharmacy follow-up likely had a negative effect on the intervention effectiveness. Future research should assess different modalities with stronger potential to reach the patient such as text and mobile calls, ensure that the technical components integrate meaningfully into workflows, and monitor follow-up from the pharmacy when patients accept vaccinations that are offered.

\section{Authors}

SAMUEL STOLPE, PharmD, MPH, National Quality Forum, Washington, DC, and NITEESH K. CHOUDHRY, MD, PhD, Harvard Medical School; Harvard T.H. Chan School of Public Health; and Center for Healthcare Delivery Sciences, Department of Medicine, Brigham and Women's Hospital, Boston, Massachusetts.

AUTHOR CORRESPONDENCE: Samuel Stolpe, PharmD, MPH, Senior Director, Quality Measurement, National Quality Forum, 1030 15th St. N.W., Ste. 800, Washington, DC 20006.

Tel.: 703.470.4741; E-mail: samuel.stolpe@mail.harvard.edu. 


\section{Effect of Automated Immunization Registry-Based Telephonic Interventions on Adult Vaccination Rates in Community Pharmacies: A Randomized Controlled Trial}

\section{DISCLOSURES}

This project was funded by Pfizer and Merck through a grant from the Pharmacy Quality Alliance. Stolpe was an employee of the Pharmacy Quality Alliance at the onset of this project and an employee of Scientific Technologies Corporation during the data collection phase of the project. Stolpe has also served on the advisory board for Merck. Choudhry has no conflicts of interest to declare.

\section{ACKNOWLEDGMENTS}

The authors acknowledge the contributions of Matthew Pickering, Brandy Altstader, and Adam Vargulick to the development of this study.

\section{REFERENCES}

1. Centers for Disease Control and Prevention. Ten great public health achievements-worldwide, 2001-2010. MMWR Morb Mortal Wkly Rep. 2011;60(24):814-18.

2. Centers for Disease Control and Prevention. Impact of vaccines universally recommended for children-United States, 1990-1998. MMWR Morb Mortal Wkly Rep. 1999;48(12):243-48.

3. Williams WW, Lu P, O'Halloran A, et al. Surveillance of vaccination coverage among adult populations-United States, 2014. MMWR Surveill Summ. 2016;65(1):1-36.

4. Resnick B, Gravenstein S, Douglas R. Empowering healthcare teams to champion culture change to improve national adult immunization rates. Symposium. Gerontologist. 2016;56 (Suppl 3):107. Available at: https://academic.oup.com/gerontologist/article/56/Suppl_3/107/2577028. Accessed July 2, 2019.

5. Koh HK, Blakey CR, Roper AY. Healthy People 2020: a report card on the health of the nation. JAMA. 2014;311(24):2475-76.

6. Rolfes MA, Flannery B, Chung J, et al. Effects of influenza vaccination in the United States during the 2017-2018 influenza season. Clin Infect Dis. February 2, 2019 [Epub ahead of print]. Available at: https://academic.oup.com/cid/advancearticle/doi/10.1093/cid/ciz075/5305915. Accessed July 2, 2019.

7. Ozawa S, Portnoy A, Getaneh H, et al. Modeling the economic burden of adult vaccine-preventable diseases in the United States. Health Affairs (Millwood). 2016;35(11):2124-32
8. Johnson DR, Nichol KL, Lipczynski K. Barriers to adult immunization. Am J Med. 2008;121(7 Suppl 2):S28-35.

9. MacDougall DM, Halperin BA, MacKinnon-Cameron D, et al. The challenge of vaccinating adults: attitudes and beliefs of the Canadian public and healthcare providers. BMJ Open. 2015;5(9):e009062.

10. Wick JY. Pharmacy-based immunization programs make an impact. Pharmacy Times. April 1, 2006. Available at: https://www.pharmacytimes. com/publications/issue/2006/2006-04/2006-04-5476. Accessed July 2, 2019.

11. Centers for Disease Control and Prevention. National early season flu vaccination coverage, United States, November 2015. Updated October 25, 2016. Available at: https://www.cdc.gov/flu/fluvaxview/nifs-estimatesnov2015.htm\#place. Accessed July 2, 2019.

12. Centers for Disease Control and Prevention. Adult immunization schedule. Available at: https://www.cdc.gov/vaccines/schedules/. Accessed July 2, 2019.

13. Centers for Disease Control and Prevention. IIS annual report. IIS data participation rates and map. 2016. Available at: https://www.cdc.gov/vaccines/ programs/iis/annual-report-iisar/rates-maps-table.html. Accessed July 2, 2019.

14. Hedden MA, Kuehl PG, Liu Y. Economic analysis of a herpes zoster vaccination program in 19 affiliated supermarket pharmacies. J Am Pharm Assoc (2003). 2014:54(4):390-96.

15. Wang J, Ford LJ, Wingate L, et al. Effect of pharmacist intervention on herpes zoster vaccination in community pharmacies. J Am Pharm Assoc (2003). 2013;53(1):46-53

16. Bryan AR, Liu Y, Kuehl PG. Advocating zoster vaccination in a community pharmacy through use of personal selling. J Am Pharm Assoc (2003). 2013;53(1):70-77.

17. Teeter BS, Garza KB, Stevenson TL, Williamson MA, Zeek ML, Westrick SC. Factors associated with herpes zoster vaccination status and acceptance of vaccine recommendation in community pharmacies. Vaccine. 2014;32(43):5749-54.

18. Brackett A, Butler M, Chapman L. Using motivational interviewing in the community pharmacy to increase adult immunization readiness: a pilot evaluation. J Am Pharm Assoc (2003). 2015;55(2):182-86.

19. Hess R. Impact of automated telephone messaging on zoster vaccination rates in community pharmacies. J Am Pharm Assoc (2003). 2013;53(2):182-87. 


\section{APPENDIX A Immunization Prompt Scripts}

\section{Patient Prompts}

Zoster only: Our records indicate that you are eligible for the shingles vaccination. Shingles vaccine is recommended for adults aged 60 and older to help protect against shingles, which can be a very painful illness that can have lasting side effects. Would you like a pharmacist to call you back to schedule your shingles shot?

Pneumococcal only: Our records indicate that you are eligible for a pneumonia vaccination. There are two types of pneumonia vaccines, with both recommended for people above the age of 65 or with certain medical conditions. Pneumonia is a serious illness that can lead to other medical complications. Would you like a pharmacist to call you back to schedule your pneumonia vaccination?

Zoster and Pneumococcal: Our records indicate that you are eligible for the shingles and pneumonia vaccinations. Would you like a pharmacist to call you back to schedule these important vaccinations?

When a patient says "Yes" then they will hear:

Great, a pharmacist will contact you to schedule this.

When a patient says "No" then they will hear:

OK, no problem. If you would like more information, please feel free to talk to one of our pharmacists.

Answering Machine Prompts

Zoster only: Our records indicate that you are eligible for the shingles vaccination. Shingles vaccine is recommended for adults aged 60 and older to help protect against shingles, which can be a very painful illness that can have lasting side effects. Please call the pharmacy to schedule your shingles vaccination.

Pneumococcal only: Our records indicate that you are eligible for a pneumonia vaccination. There are two types of pneumonia vaccines, with both recommended for people above the age of 65 or with certain medical conditions. Pneumonia is a serious illness that can lead to other medical complications. Please call the pharmacy to schedule your pneumonia vaccination.

Zoster and Pneumococcal: Our records indicate that you are eligible for the shingles and pneumonia vaccinations. Please call the pharmacy to schedule these important vaccinations.

\begin{tabular}{|c|c|c|}
\hline APPENDIX B & \multicolumn{2}{|c|}{$\begin{array}{l}\text { Baseline Characteristics of Intervention } \\
\text { Patients by Call Completion }\end{array}$} \\
\hline \multirow[b]{2}{*}{ Characteristics } & \multicolumn{2}{|c|}{ Intervention Call Status ${ }^{a}$} \\
\hline & Incomplete & Completed \\
\hline Number (\%) & $7,343 \quad(66.6)$ & $3,666(33.3)$ \\
\hline Age, years, mean (SD) & $62.0(14.4)$ & $65.7(13.0)$ \\
\hline Female, \% & 54.2 & 62.0 \\
\hline $\begin{array}{l}\text { Median income in ZIP code, } \\
\text { mean (SD), \$ }\end{array}$ & $67,588 \quad(17,583)$ & $65,878 \quad(16,144)$ \\
\hline $\begin{array}{l}\text { Black race in ZIP code, } \\
\text { mean (SD), \% }\end{array}$ & $5.0(0.1)$ & $4.4(0.1)$ \\
\hline $\begin{array}{l}\text { Education in ZIP code, } \\
\% \text { undergrad or higher (SD) }\end{array}$ & $27.2(12.3)$ & $25.5(12.3)$ \\
\hline$\leq 0.005$ & & \\
\hline
\end{tabular}

